\title{
Temperature profile of IR blocking windows used in cryogenic X-ray spectrometers
}

\author{
S. Friedrich, T. Funk, O. Drury, and S. E. Labov
}

This article was submitted to

SPIE Meeting

San Diego, CA

July 30 - August 4, 2000

\section{August 8, 2000}

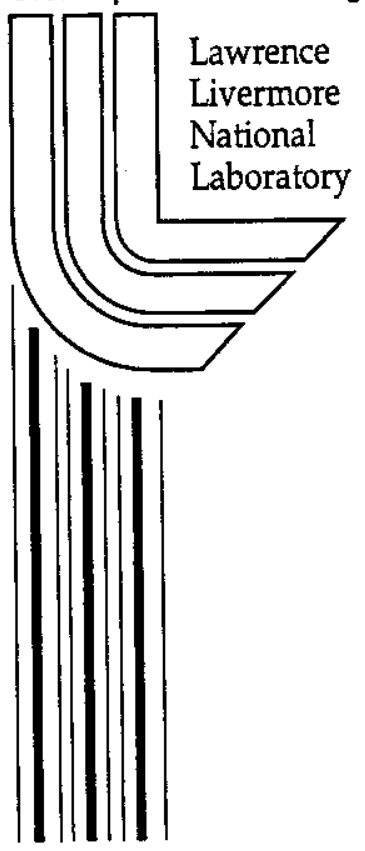




\section{DISCLAIMER}

This document was prepared as an account of work sponsored by an agency of the United States Government. Neither the United States Government nor the University of California nor any of their employees, makes any warranty, express or implied, or assumes any legal liability or responsibility for the accuracy, completeness, or usefulness of any information, apparatus, product, or process disclosed, or represents that its use would not infringe privately owned rights. Reference herein to any specific commercial product, process, or service by trade name, trademark, manufacturer, or otherwise, does not necessarily constitute or imply its endorsement, recommendation, or favoring by the United States Government or the University of California. The views and opinions of authors expressed herein do not necessarily state or reflect those of the United States Government or the University of California, and shall not be used for advertising or product endorsement purposes.

This is a preprint of a paper intended for publication in a journal or proceedings. Since changes may be made before publication, this preprint is made available with the understanding that it will not be cited or reproduced without the permission of the author.

This report has been reproduced

directly from the best available copy.

Available to DOE and DOE contractors from the

Office of Scientific and Technical Information

P.O. Box 62, Oak Ridge, TN 37831

Prices available from (423) 576-8401

http://apollo.osti.gov/bridge/

Available to the public from the

National Technical Information Service

U.S. Department of Commerce 5285 Port Royal Rd.,

Springfield, VA 22161

http://www.ntis.gov/

OR

Lawrence Livermore National Laboratory

Technical Information Department's Digital Library

http://www.llnl.gov/tid/Library.htmI 


\title{
Temperature profile of IR blocking windows used in cryogenic X-ray spectrometers
}

\author{
S. Friedrich ${ }^{2, b}$, T. Funk $k^{b}$, O. Drury ${ }^{a}$, S. E. Labov ${ }^{a}$ \\ Lawrence Livermore National Laboratory, L-418, P.O. Box 808, Livermore, CA 94551 \\ Lawrence Berkeley National Laboratory, MS 6-2100, Berkeley, CA 94720
}

\begin{abstract}
Cryogenic high-resolution X-ray spectrometers are typically operated with thin IR blocking windows to reduce radiative heating of the detector while allowing good $\mathbf{x}$-ray transmission. We have estimated the temperature profile of these IR blocking windows under typical operating conditions. We show that the temperature in the center of the window is raised due to radiation from the higher temperature stages. This can increase the infrared photon flux onto the detector, thereby increasing the IR noise and decreasing the cryostat hold time. The increased window temperature constrains the maximum window size and the number of windows required. We discuss the consequences for IR blocking window design.
\end{abstract}

Keywords: IR blocking windows, IR noise, cryogenic detectors, superconducting tunnel junctions

\section{INTRODUCTION}

Cryogenic $\mathrm{x}$-ray spectrometers, i.e. spectrometers with a detector temperature below $\mathrm{IK}$, have received much interest over the last decade because of their superior energy resolution compared to conventional semiconductor-based detectors $[1,2]$. Several technologies are being developed which have demonstrated an energy resolution between 1 and $2 \mathrm{eV}$ FWHM for soft $\mathrm{x}$-rays [3,4] and between 4.5 and $13 \mathrm{eV}$ at $6 \mathrm{keV}[5-8]$.

One concern in spectroscopy applications with cryogenic detectors is how to efficiently transmit $x$-rays from an external source onto the detector inside the cryostat while shielding the detector from IR radiation. IR shields reduce the heat load into the low temperature stage of the cryostat and eliminate excess noise due to the absorption of IR photons. Typically, the shields consist of several thin AI IR blocking windows, which are sometimes supported by a parylene or polyimid film and/ or a $\mathrm{Ni}$ grid for increased stability. The filter design then involves a trade-off between maximizing the $x$-ray transmission and acceptance angle and minimizing the IR photon flux.

We are presently building a cryogenic spectrometer for synchrotron-based $\mathrm{x}$-ray fluorescence applications. It uses a twostage adiabatic demagnetization refrigerator (ADR) with a base temperature below $0.1 \mathrm{~K} \mathrm{[9]}$. The cryogenic detector is held at the end of a cold finger that protrudes into the sample chamber. The distance between the detector and the room temperature fluorescence target is about $2 \mathrm{~cm}$. The cold finger uses radiation shields attached to the cryostat's liquid $\mathrm{N}_{2}$ and liquid He-cooled stages with IR blocking windows at $77 \mathrm{~K}, 4.2 \mathrm{~K}$ and $0.2 \mathrm{~K}$. The small distance between windows increases the radiative heat load because of an increased solid angle of illumination and multiple photon reflections. This raises the temperature in center of the window above the temperature of the window frame. Here we present calculations to estimate the temperature profile of the windows under typical operating conditions. We show how the $i R$ heat load of the detector depends on the window parameters, and provide guidelines on how to optimize the window design.

\section{MODEL}

Consider a circular window with thickness $d$ and radius $R$, which is heated by radiation from a higher temperature stage and whose temperature at the perimeter is held at the temperature $T_{0}$ of the metal shield it is mounted to. The window is sufficiently thin so that the temperature is constant along its thickness, and there is no angular dependence of the temperature because of the rotational symmetry of the window. In steady state, the temperature of the IR window then only depends on its radial coordinate $r$. The temperature distribution is governed by the heat flow equation [10] 


$$
\frac{1}{r} \frac{\partial}{\partial r}\left(K(T(r)) \cdot r \frac{\partial T}{\partial r}\right)=\frac{\sigma\left(2 \varepsilon T(r)^{4}-a_{e f f} T_{e x t}^{4}\right)}{d} \quad \text { with } T(R)=T_{0} \text { and }\left.\frac{\partial T}{\partial r}\right|_{r=R}=0
$$

where $\mathrm{K}$ is the thermal conductivity of the window, $\sigma=5.610^{-12} \mathrm{WK}^{-4} \mathrm{~cm}^{-2}$ is the Stefan-Boltzmann constant, $\varepsilon$ is the emissivity of the window and $a_{e f f}$ is the effective absorbance of the window which takes into account multiple photon reflections. Equation (1) describes how heat input from an external source at temperature $T_{\text {ext }}$ is balanced by conductive and radiative cooling of the window. Note that the radiative terms enter as heat sources/ sinks per unit volume, so that their explicit dependence on the window area falls out of the equation. The factor of 2 accounts for the fact that the window can emit radiation into both directions, while it is heated only from one side. The second boundary condition arises from symmetry considerations and ensures that the solutions of the temperature profile do not diverge at the origin.

We now apply this model to the IR blocking windows used in our experiments. They consist of a $200 \AA$ high-reflectivity Al film on top of $1000 \AA$ of parylene $\left(\mathrm{C}_{2} \mathrm{H}_{8}\right)$ fabricated by Lebow Company [11]. The Al is thermally evaporated onto the parylene film so that both films are in intimate thermal contact. The thermal conductivity of $\mathrm{Al}$ is given by the WiedemannFranz law $K=L T / \rho$, where $L=2.410^{-8}{\mathrm{~W} \Omega K^{-2}}^{-2}$ the Lorenz number, and $\rho$ is the electrical resistivity of the film. At room temperature, $\rho_{\mathrm{Al}}=2.65 \mu \Omega \mathrm{cm}$, while a low temperatures $\rho_{\mathrm{Al}}$ is reduced by the by the residual resistivity ratio RRR which depends on the film purity. For a conservative estimate of the thermal conductivity $K$ we make the assumption $R R R=2$ and

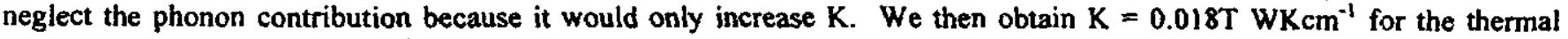
conductivity of the Al film. The Wiedemann-Franz approximation we use assumes low values for $K$ even for superconducting Al below $1.2 \mathrm{~K}$ where thermal energy is carried by phonon transport only [12]. The thermal conductivity of the parylene film at room temperature is only $1.2 \cdot 10^{-2} \mathrm{WK}^{-1} \mathrm{~cm}^{-1}[11]$, and it decreases at lower temperatures. To our knowledge, no data on the thermal conductivity of parylene at temperatures below $1 \mathrm{~K}$ have been published, but we expect it to be of order $10^{-4} \mathrm{WK}^{-1} \mathrm{~cm}^{-1}$, comparable to that of other hydrocarbons [12]. At all temperatures the $\mathrm{Al}$ film thus determines the thermal conductivity of the IR blocking window.

For closely spaced parallel windows, the effective absorbance $a_{\text {eff }}$ is increased due to multiple photon reflections in the cavity between the windows. Photons impinging on one of the windows have a probability $\varepsilon$ to be absorbed, a probability $\tau$ to be transmitted and a probability $1-\varepsilon-\tau$ to be reflected. For our highly reflective $200 \AA \mathrm{Al}$ films, $\varepsilon=0.04$ and $\tau=0.04[11,13]$, and photons can be reflected many times before being absorbed by either one of the windows or the detector. For an average of $\mathrm{N}$ reflections, the effective absorbance in one of the windows is given by

$$
a_{e f f}=\sum_{n=0}^{N} \varepsilon(1-\varepsilon-\tau)^{2 n}=\varepsilon \frac{(1-\varepsilon-\tau)^{2(N-1)}-1}{(1-\varepsilon-\tau)^{2}-1}
$$

A lower limit to the absorbance is $a_{e f f}=\varepsilon$ for the case $N=0$. This case is realized for windows whose spacing $D$ is much larger than the window diameter $2 R$, which makes multiple reflections unlikely. An upper limit is realized for parallel windows spaced much closer than their diameter. In this case $N=\infty$ and $a_{e f f} \approx \varepsilon / 2(\varepsilon+\tau)$. In this limit photons are reflected sufficiently often to distribute their energy evenly between the two windows. For our $200 \AA$ Al films, where $\varepsilon \approx \tau$, we find $a_{\text {eff }}=0.25$. This means that about $1 / 4$ of the photons is eventually absorbed in each window and $1 / 4$ of the photons is transmitted through each window. The geometry discussed in the remainder of this paper is an intermediate case where the window spacing $D$ is comparable to the window diameter $2 R$. In this case, many of the photons striking one window will come from significant off-axis angles, and multiple reflections will cause the photons to strike the filter frame after"a few reflections. We make the crude approximation of an average number of reflections $N=2 R / D$. The effective absorbance is then given by $a_{\text {eff }}=(1+R / D) \varepsilon$. The value of the effective transmission coefficient $\tau_{\text {eff }}$ is calculated analogously.

We assume that each window is heated by radiation from all higher temperature stages. Then $\mathrm{a}_{\mathrm{eff}} \mathrm{T}_{\mathrm{ext}}{ }^{4} \Rightarrow \Sigma_{\mathrm{a}_{\mathrm{i}}} \mathrm{T}_{\mathrm{i}, \mathrm{ext}}{ }^{4}$ in equation (1), with the summation index $i$ labeling all higher temperature stages. The contribution to the heat input from each of these stages is weighted by the absorbance $a_{3}=\varepsilon$ for radiation from the closest stage, by $a_{2}=\tau a_{\text {eff }}$ for radiation from the next warmer stage (relevant for the $0.2 \mathrm{~K}$ and $4 \mathrm{~K}$ window only) and by $a_{3}=\tau \tau_{\text {eff }} \hat{a}_{\text {eff }}$ for radiation from the outermost stage (here relevant only for heating of the $0.2 \mathrm{~K}$ window by room temperature radiation). A more detailed calculation, which would involve a Monte-Carlo ray tracing simulation that includes absorption, transmission and reflection at each surface, is beyond the scope of this paper. 


\section{RESULTS AND DISCUSSION}

An approximate analytic solution of the temperature profile can be obtained if the $T(r)^{4}$ term in equation $(l)$ is neglected and if the thermal conductivity of the window is assumed to be constant. In this case

$$
T(r)=T_{0}+\frac{a_{e f f} \sigma T_{e x t}^{4}\left(R^{2}-r^{2}\right)}{4 K d}
$$

The first approximation is valid for the low temperature windows at $4.2 \mathrm{~K}$ and $0.2 \mathrm{~K}$ where radiative cooling is negligible. The second approximation is usually not satisfied, but for a conservative estimate of $K=K\left(T_{0}\right)$ equation (3) still provides an upper limit to the temperature that can be calculated easily for different window designs and geometries. We will discuss later which constant value of $K$ to assume for a better estimate of the temperature.

In general, the temperature distribution of equation (3) is not valid and the boundary value problem (1) must be solved numerically. We have reduced this problem to a first-order integral-differential equation through multiplication by $\mathrm{r}$ and integration over $r$. The resulting right-hand-side integral is then solved numerically. Starting at a constant shield temperature, the temperature profile of the window is approximated iteratively using a fourth-order Runge-Kutta algorithm until self-consistency is achieved. We have confirmed that the numerical solution agrees with the analytic solution (3) for the case of constant thermal conductance and negligible radiative cooling.

Figure 1 shows the temperature profile of the three windows at $77 \mathrm{~K}, 4.2 \mathrm{~K}$ and $0.2 \mathrm{~K}$ for a window spacing $\mathrm{D}=0.5 \mathrm{~cm}$ and two different window diameters. We show cases where the higher temperature windows are larger than the low temperature windows as required for optimum solid angle coverage. The dashed line is the result of the analytic solution when radiative cooling is neglected and the thermal conductivity is given by $K=K\left(T_{0}\right)$ where $T_{0}$ is the temperature of the shield. Figure 1 shows that radiation from the higher temperature stages can heat the center of each IR blocking window well above the temperature of the shield it is mounted to. As expected, smaller windows are heated less because a smaller window has a higher thermal conductance than a bigger one with the same conductivity. The deviation of the temperature profile in the simulation from the quadratic form of equation (3) is due to the increased thermal conductivity of the window at higher temperatures according to the Wiedemann-Franz law. Radiative cooling only contributes significantly for large windows on the $77 \mathrm{~K}$ stage, and is completely negligible for the low temperature windows.
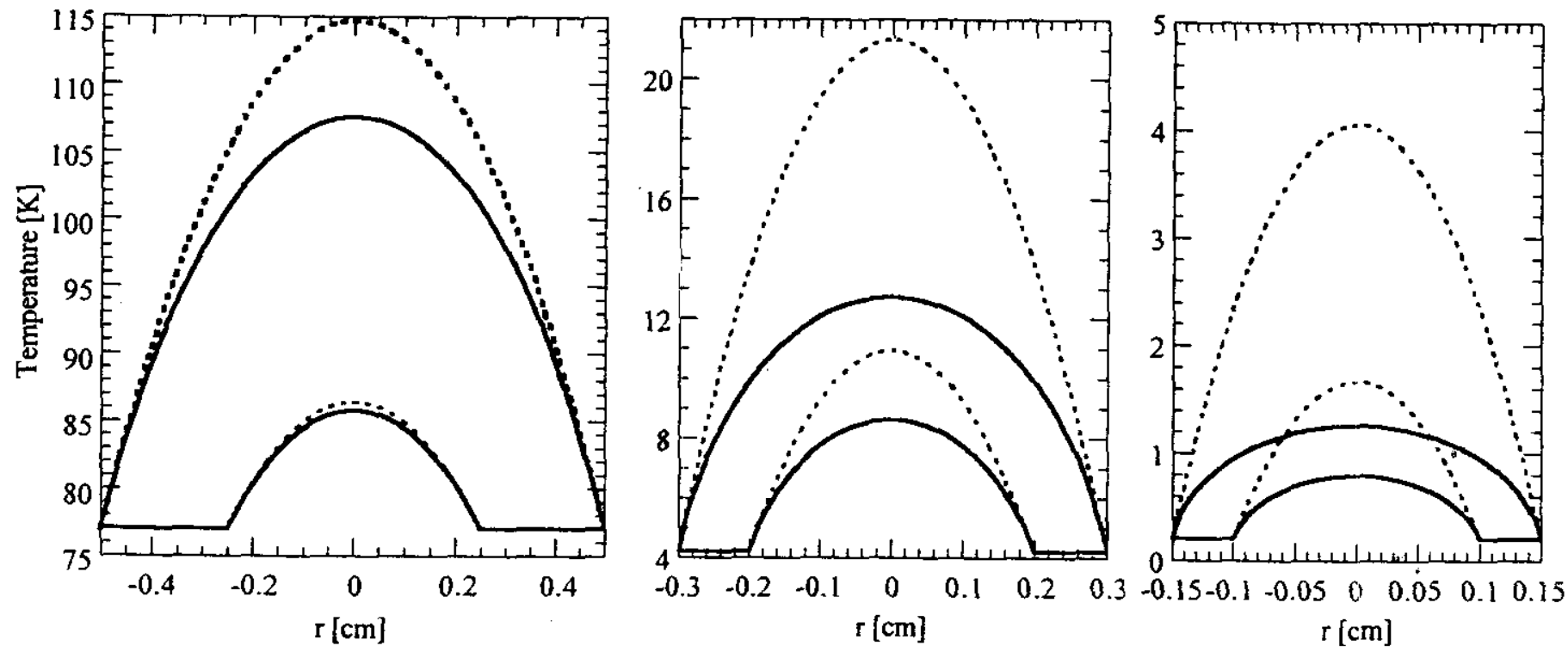

Figure 1: Temperature profile of the IR blocking windows at the liquid $\mathrm{N}_{2}$-cooled shield at $\mathrm{T}_{0}=77 \mathrm{~K}$, at the liquid He-cooled shield at $T_{0}=4.2 \mathrm{~K}$ and at the cold stage shield at $T_{0}=0.2 \mathrm{~K}$ (solid lines). The dashed lines are approximations neglecting radiative cooling and assuming a constant thermal conductance $\mathrm{K}\left(\mathrm{T}_{0}\right)$ as in equation (3). They constitute an upper limit to the temperature profile. 


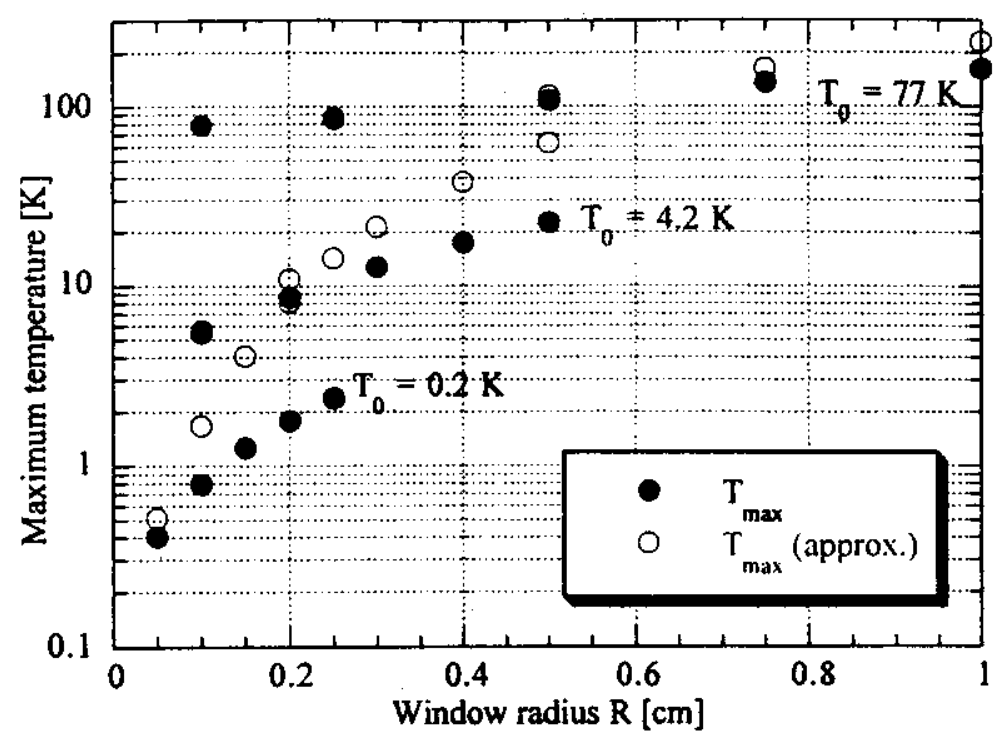

Figure 2: Peak temperature of the IR blocking window as a function of size for windows mounted to the $77 \mathrm{~K}$ shield (top rows), the $4.2 \mathrm{~K}$ shield (middle) and the cold stage shield (bottom). The solid circles are the results of the full numerical simulation, and the open circles are worst case approximations according to equation (3) for constant thermal conductivity $K\left(T_{0}\right)$ and without radiative cooling.

The peak temperature in the center of the window as function of window radius is shown in figure 2 . We find that even a comparably small window with $5 \mathrm{~mm}$ diameter on the $0.2 \mathrm{~K}$ cold stage will be heated to well above $2 \mathrm{~K}$ if only two IR blocking windows on the higher temperature stages are used. Figure 2 provides some guidance which window size to choose to keep the window below a certain temperature.

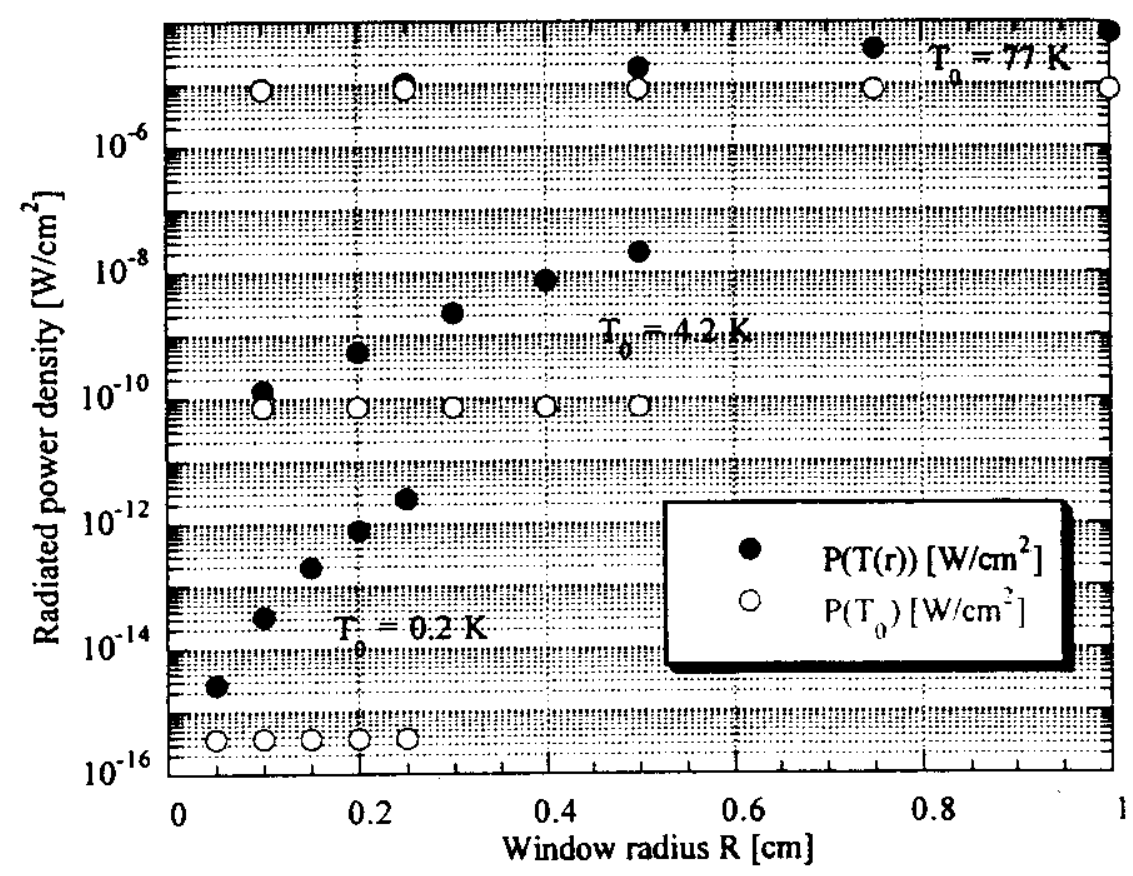

Figure 3: Radiated power per unit area from each window for the temperature profiles calculated above (solid circles). For comparison, the open circles show the radiated power for the ideal case where the entire window is held constant at the temperature $T_{0}$ of the shield it is mounted to. 
Ultimately, we are interested in the temperature distribution in the window because it determines the radiated power each window emits, which could heat the cold stage and cause excess noise in the detector. In figure 3 we show the total radiated power density from each window. Because of the strong $\mathrm{T}^{4}$ dependence of the Stefan-Boltzmann law, even small increases in the window temperature can increase the radiated power by several orders of magnitude. For windows sufficiently small the radiated power can be reduced considerably. Figure 3 does, however, show that it is not feasible to operate a cryogenic detector with only two closely spaced IR blocking windows, unless they are very small or one is willing to accept the increased IR flux and consequently a decrease in detector resolution and cryostat hold time.

In order to understand which radiative source dominates the heating of each window, we can compare the magnitude of the heat input terms in the sum $\Sigma a_{i} T_{i, e x t}{ }^{4}$. We find that for $200 \AA \mathrm{Al}$ windows the dominant heat source for all windows and for the detector is the fraction of room temperature radiation transmitted through the higher temperature windows. To verify that the increased temperature of the $77 \mathrm{~K}$ window does not significantly affect the temperature profile of the lower stage windows, we have simulated the temperature profile at each window successively, with the exact temperature profile of each outer stage determining the radiative load into the next colder stage. The results of this simulation, including the temperature profile of the $77 \mathrm{~K}$ window used, are shown in figure 4 . For comparison we also show the temperature profile of the $4 \mathrm{~K}$ stage and the $0.2 \mathrm{~K}$ stage windows calculated with the assumption that the window on the liquid $\mathrm{N}_{2}$-cooled stage is held constant at $T_{0}=77 \mathrm{~K}$ (dashed lines). The differences are marginal, certainly within the uncertainty due to the errors in the thermal conductivity of the Al. The increased temperature of the outer windows does not significantly increase the heat load onto the inner windows, which is still dominated by room temperature radiation.

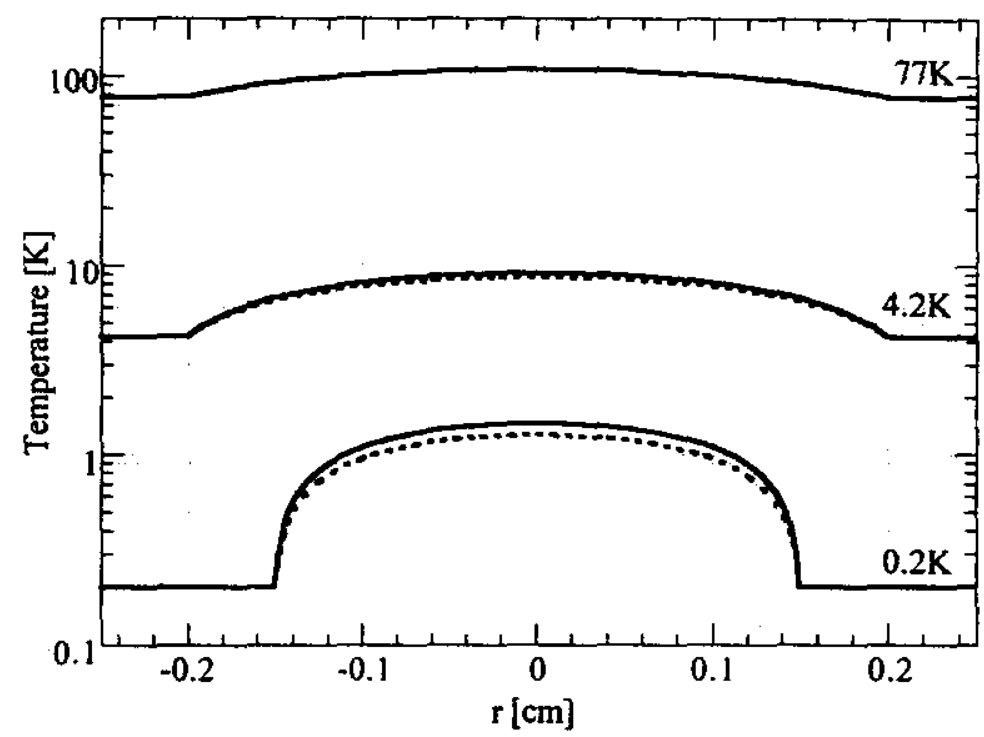

Figure 4: Effects of the increased temperature of the $77 \mathrm{~K}$ window on the temperature profile of the colder stages. The dashed profiles are based on the assumption of a constant temperature $\mathrm{T}_{0}=77 \mathrm{~K}$ of the liquid $\mathrm{N}_{2}$-cooled window. The solid profiles take into account the increased radiation from the heated $77 \mathrm{~K}$ window. The differences are very small.

There are several ways to further reduce the IR load onto the cold stage and the detector. One is to mount a small tube on the $77 \mathrm{~K}$ stage in front of window, thereby reducing the solid angle over which room temperature radiation falls onto the windows. This has proved very effective in our earlier cryostats. However, such a tube defeats the main purpose of a cold finger, which is to bring the cryogenic detector close to the fluorescent sample positioned in an experimental chamber outside the cryostat. Another technique is to tilt the windows by about 10 degrees relative to one another. This reduces the effects of multiple photon reflections, which is, however, not all that strong in our geometry. A good way to absorb photons in the cavity between the windows is to coat the face of the $4.2 \mathrm{~K}$ window hoider towards the $77 \mathrm{~K}$ stage black. This absorbs photons without increasing the emissivity of the $4.2 \mathrm{~K}$ stage towards the cold stage. The most effective improvement can be obtained with a thicker Al window in at least one of the stages. A $1000 \AA \mathrm{Al}$ film would have a transmission of $\tau=10^{-5}$ for room temperature radiation, more than three orders of magnitude less than the $200 \AA \mathrm{Al}$ films with $\tau=0.04$ we presently use. The optimum Al thickness depends on a trade-off between the $x$-ray transmission required and the maximum radiative load 
the detector can operate with. One can also add a second thin window on the $77 \mathrm{~K}$ or the $4.2 \mathrm{~K}$ stage, thereby reducing the radiative heat load into the cold stage by more than an order of magnitude. Measuring the reduction in the noise spectrum of the cryogenic detector can be used to test the effectiveness of these modifications. Experimental determination of the optical and electrical characteristics of the Al film, in particular the residual resistance ratio and the transmissivity, will also allow a better quantification the calculations.

Finally, we provide an empirical formula how to better estimate an effective constant thermal conductance of a window, if only a rough estimate of the temperature profile according to equation (3) is desired. We have fit the temperature profile of equation (3) to the exact temperature profiles from the numerical simulation. An example of such a fit is shown in figure 5a. The solid line is the temperature profile numerically calculated using the full expression in equation (1) for two windows at the $4.2 \mathrm{~K}$ stage with different radii. The dashed line is a least square fit to this profile using equation (3) with $\mathrm{K}$ as the variable parameter. The constant thermal conductivity of the fit overestimates the exact value in the outer parts of the window, and underestimates it in the center. This is because the actual thermal conductivity of the window assumes its lowest value at the low temperature frame and increases towards the higher temperature center.
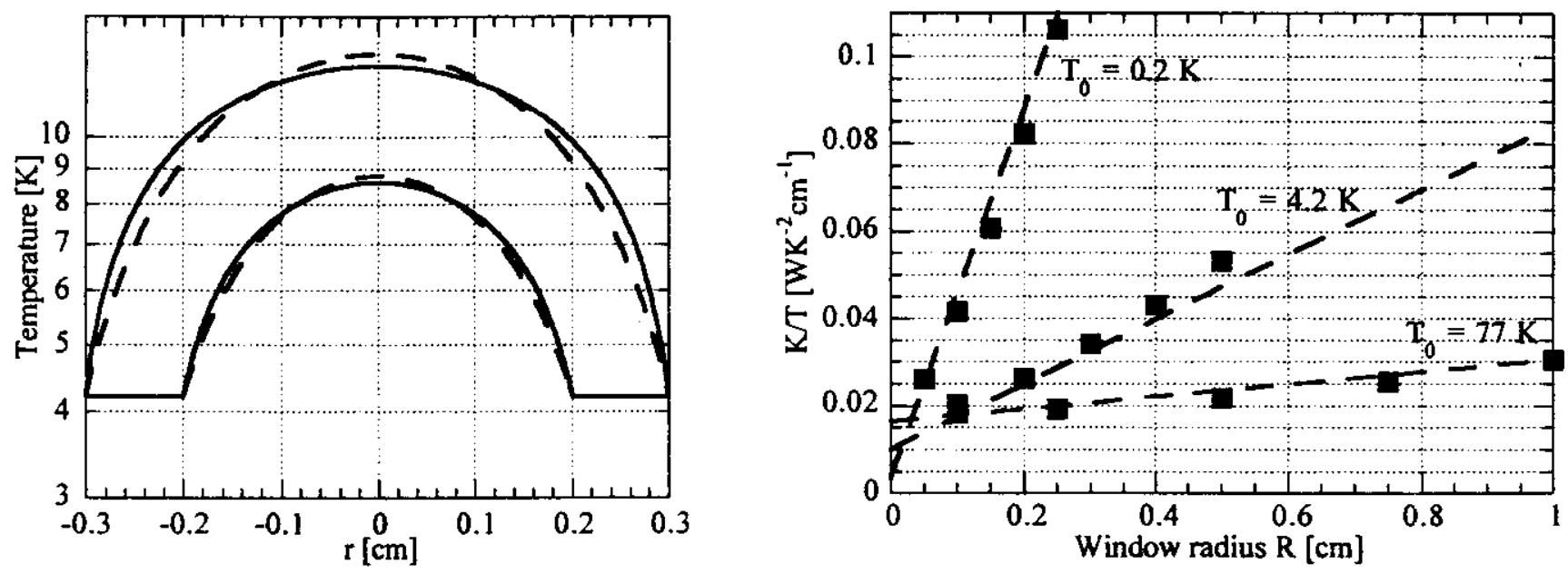

Figure 5: a) Temperature profile of the $4.2 \mathrm{~K}$ stage windows (solid lines) and least squares fit using equation (3) with $\mathrm{K}$ as fitting parameter (dashed lines). b) Empirical fit to the optimum $\mathrm{K} / \mathrm{T}_{0}$ value according to equation (4).

The least-square fit values of $\mathrm{K} / \mathrm{T}_{0}$ for the numerical temperature distributions of windows with different radii $R$ are shown in figure $5 \mathrm{~b}$. The dashed lines follow the empirical relation

$$
\frac{K\left(R, T_{0}\right)}{T_{0}}=0.0069+0.005 \log _{10}\left(T_{0}\right)+R \cdot 0.169 T_{0}^{-0.57}
$$

Equation (4) provides a rough estimate of the effective thermal conductance $K$ for an $A$ l window with radius $R$ and a residual resistance ratio of 2 mounted to a shield at temperature $T_{0}$. It can provide guidance on whether the shielding of a certain set of IR blocking windows is clearly sufficient, clearly insufficient or whether a more detailed numerical calculation is needed.

\section{SUMMARY}

We have estimated the temperature profile of IR blocking windows used in cryogenic spectrometers under typical operating conditions. We find that the center of the window can be heated well above the temperature of the shield it is mounted to. Window heating does, however, not increase the radiative load onto the detector significantly for typical geometries. This heat load is usually dominated by the faction of room temperature radiation transmitted through the windows. The dominant cooling mechanism of the window is conductive cooling through the Al coating. We have provided guidelines for an estimate of the temperature profile of an IR blocking window and the resulting radiative heat load. More precise calculations of the temperature profile require experimentally determined values of the optical and electrical characteristics of the window. 


\section{ACKNOWLEDGEMENTS}

We thank Dan McCammon, Scott Porter and Ed Graper for useful and stimulating discussions. This work was performed under the auspices of the U.S. Department of Energy by University of California Lawrence Livermore National Laboratory under contract No. W-7405-Eng-48. Funding was provided by NASA under UV Detector Development Program grant NAG5-4137.

\section{REFERENCES}

1. N.E. Booth, D.J. Goldie, "Superconducting particle detectors", Supercond.Sci. Technol. 9, pp 493-516, 1996

2. D. Twerenbold, "Cryogenic particle detectors", Rep. Prog. Phys. 59, pp 349-426, 1996

3. S. Kraft, P. Verhoeve, A. Peacock, N. Rando, D.J. Goldie, R. Hart, D. Glowacka, F. Scholze, G. Ulm, "On the factors governing the energy resolution of Ta-based superconducting tunnel junctions", J. Appl. Phys 86, pp 7189-7191, 1999

4. S. Friedrich, J.B. le Grand, L.J. Hiller, J. Kipp, M. Frank, S.E. Labov, S.P. Cramer, A.T. Barfknecht, "High-Resolution Tunnel Junction Extreme Ultraviolet Detectors Limited by Quasiparticle Counting Statistics", IEEE Trans. Appl. Superconductivity 9, pp 3330-3333, 1999

5. K.D. Irwin, G.C. Hilton, J.M. Martinis, S. Deiker, N. Bergren, S.W. Nam, D.A. Rudman, D.A. Wollman, "A Mo-Cu superconducting transition-edge microcalorimeter with $4.5 \mathrm{eV}$ energy resolution at $6 \mathrm{keV}^{\prime \prime}$, Nucl. Inst. Meth. A444, pp $184-187,2000$

6. A. Alessandrello, J.W. Beeman, C. Brofferio, O. Cremonesi, E. Fiorini, A. Giuliani, E.E. Haller, A. Monfardini, A. Nucciotti, M. Pavan, G. Pessina, E. Previtali, L. Zanotti, "High Energy Resolution Bolometers for Nuclear Physics and X-Ray Spectroscopy", Phys. Rev. Lett. 82, pp 513-515 (1999)

7. G. Angloher, B. Beckhoff, M.Buhler, F. von Feilitzsch, T. Hertrich, P. Hettl, J. Höhne, M. Huber, J. Jochum, R.L. Mössbauer, J. Schnagl, F. Scholze, G. Ulm, "Development of superconducting tunnel junction detectors for highresolution X-ray spectroscopy", Nucl. Inst. Meth. A444, pp 214-219, 2000

8. D.E. Prober, to be published in "Proceedings of the Conference on Space Astrophysics Detectors and Detector Technology", Baltimore, 2000

9. C. Hagmann, P.L. Richards, "Two-stage magnetic refrigerator for astronomical applications with reservoir temperatures above 4K", Cryogenics 34, pp 221-226, 1994

10. See for example H.S. Carslaw, J.C. Jaeger, "Conduction of Heat in Solids", 2nd ed., Oxford Science Publications, 1959

11. Lebow Company, Goleta, CA, (805) 964-7117; Ed Graper (private communication)

12. "Thermophysical Properties of Matter", The TPRC Data Series, Y.S. Touloukian, C.Y. Ho (ed.), Plenum Press, New York, 1970

13. G.K. White, "Experimental Techniques in Low-Temperature Physics", 3rd ed., Oxford Science Publications, 1979 
